\title{
Utilización de balones con diferentes masas en la precisión y el desplazamiento máximo aéreo del remate en fútbol infantil
}

\author{
The precision and maximal air displacement in the shoot at goal \\ in infant football with different balls masses
}

\author{
Patricia Alejandra Polero Campione \\ José Fernández ${ }^{2}$ \\ Juan Manuel Meyer ${ }^{2}$ \\ Bruno Mendoza 2 \\ Renata Luísa Bona ${ }^{1}$
}

\section{RESUMEN}

Objetivo: Comparar la diferencia entre dos balones de diferentes masas (reglamentario y voleibol) en la ejecución del gesto de remate e identificar cuál es más adecuado para niños de 60 a 84 meses. Método: Para evaluar el gesto del remate fueron realizadas tres pruebas de precisión - I) prueba de precisión en portería reglamentaria de fútbol para niños con obstáculo, II) prueba de precisión en mini portería, III) prueba de precisión con portería reglamentaria de fútbol para niños; Y una prueba de desplazamiento máximo - prueba de desplazamiento del balón. Todas las pruebas fueron realizadas con dos diferentes balones. Resultados: Se encontraron diferencias para la prueba de precisión en portería reglamentaria de fútbol para niños con obstáculo y para el desplazamiento máximo. Conclusión: Las diferencias encontradas indican que la masa del balón podría afectar la práctica de fútbol para niños. Aplicación práctica: Es importante evitar posibles lesiones durante la práctica de fútbol para niños, dicho cuidado en la formación demanda la implementación de los correctos estímulos utilizados en el proceso de aprendizaje.

\section{PALABRAS-CLAVES}

Deporte; Infante; Prevención; Aprendizaje; Materiales.

\footnotetext{
${ }^{1}$ Laboratorio de Biomecánica y Análisis del Movimiento, Universidad de la Republica Uruguay, Centro Universitario de Paysandú, Paysandú, Uruguay.

universidad de la Republica Uruguay, Centro Universitario de Paysandú, Instituto Superior de Educación Física, Paysandú, Uruguay.
} 


\section{ABSTRACT}

Aim: To compare the precision and maximal air displacement in the shoot at goal in infant football with different balls masses (soccer ball and volleyball) and to identify which ball is more suitable for children between 60 and 84 months old. Methods: For this goal three precision test were utilizided - I) regulatory space football precision for children test with obstacles, II) mini space football precision, III) regulatory space football precision for children; and maximal displacement test - ball displacement test. All tests were performed with the two different balls. Results: Differences in tasks performed with the different balls were found in two tests: regulatory space football precision for children test with obstacles and for ball displacement test. Conclusion: We believe these differences could affect the children soccer practice. Practical application: To prevent injuries is important in children soccer practice, for this reason is important the correct triggers implementation in learning process.

\section{KEYWORDS}

Sports; Child; Prevention; Learning; Materials. 


\section{INTRODUCCIÓN}

El fútbol es un deporte que vincula a grandes masas ya sea como practicantes o como espectadores. En muchos países se comienza a practicar desde edades muy tempranas. La práctica del fútbol, dota al cerebro de los niños de amplias destrezas y habilidades, no sólo motrices, sino también intelectuales (Benedek, 2006; Castelo, 1999). A través de su práctica, los niños entran en contacto con estímulos motrices, que generan un amplio acervo motor debido a su complejidad coordinativa. Muchas veces, dicha práctica genera lesiones, principalmente en miembros inferiores y en el cráneo. El grado de lesiones padecidas durante las etapas de crecimiento y maduración, condicionan probables consecuencias para los años venideros en la integridad física de las personas (Rössler et al., 2016).

O'Kane y colaboradores 2014, encontró que cabecear el balón representó el 30,5 \% de las conmociones cerebrales detectadas, en jugadoras de fútbol de secundaria. En los partidos se dieron el 30,6 \% de las conmociones cerebrales entre los jóvenes, y el 25,3\% entre las adolescentes (O'Kane et al., 2014).

Además, un estudio realizado en República Checa y Suiza, con jugadores de 7 a 12 años durante dos temporadas, registró un total de 417 lesiones de un total de 15 niños. La mayoría (76,3\%) de las lesiones se localizan en los miembros inferiores. Lesiones en las articulaciones y los ligamentos comprenden el $30,5 \%$, contusiones $22,5 \%$, lesiones musculares y tendinosas $18,5 \%$, y las fracturas y lesiones óseas $15,4 \%$ de todas las lesiones. Además, $23,7 \%$ de las lesiones llevaron a más de 28 días de ausencia de participación en el deporte (Rössler et al., 2016). La incidencia de lesiones observadas son menores en comparación con los estudios de jugadores juveniles. Los niños presentan una proporción relativamente alta de fracturas, estrés del hueso y lesiones de las extremidades superiores. Los estudios muestran la importancia de desarrollar un programa de prevención de lesiones por edad (Rössler et al., 2016).

El fútbol es exclusivo en comparación con otros deportes, en el que los participantes usan deliberadamente su cabeza para golpear el balón. Las lesiones en la cabeza durante los partidos de fútbol, son generalmente el resultado del contacto directo contra la cabeza de otro jugador, contra la rodilla, contra el suelo, o contra el balón. Existe la posibilidad de modificar algunas medidas, como el balón para los niños, o reglas de juego para prevenir lesiones y lograr proteger la salud de los jugadores de fútbol, haciendo énfasis en la protección de la cavidad craneana. Es posible tomar varias medidas para evitar el desarrollo de las conmociones cerebrales en el fútbol (Levy et al., 2012; Less et al., 2010).

Siendo conscientes de la gran importancia que conlleva la formación integral de las personas, y más específicamente la de los niños, quienes presentan características de aprendizaje muy particulares, se entiende que se requiere de un cuidado especial en la formación de base. Esto contribuirá en un propicio desarrollo físico-motriz y cognitivo (Babkes y Weiss, 1999; Verburgh et al., 2014). Este estudio fue realizado para comparar la diferencia en la ejecución de algunos gestos con dos balones de diferentes masas (reglamentario y voleibol) e identificar cuál es más adecuado para niños de 60 a 84 meses, en la ejecución del gesto de remate y para medida de máxima distancia. Nuestra hipótesis es que el balón con menor masa al del balón reglamentario de fútbol infantil para niños entre 60 a 84 meses de edad, será más adecuado en la realización correcta del gesto de remate.

\section{MATERIALES Y MÉTODOS}

\section{Muestra}

Participaron del estudio 21 niños varones de entre 60 y 84 meses de edad. Todos practicantes activos del deporte fútbol en el club Estudiantil Sanducero F.C., con una continuidad de prácticas de tres veces por semana, una hora de actividad cada día. Ninguno de los participantes presentaban patologías musculoesqueléticas o neurológicas que perjudiquen la función normal del aparato locomotor. Todos los adultos responsables de los niños que participaron firmaron un consentimiento informado.

\section{Protocolos}

Las pruebas se realizaron en la cancha de fútbol donde los niños practican semanalmente, en el horario habitual, con su entrenador presente. Los par- 
ticipantes no sabían que estaban pateando con diferentes balones, (ni cuál era el objetivo del estudio). Las cuatro pruebas (tres de precisión y una de desplazamiento) fueron pensadas teniendo en cuenta consignas muy comunes en la enseñanza del deporte, en un período de tres días, durante una hora cada día. Se plantearon a modo de juego, desafiando a los niños a lograr un objetivo.

El primer día se realizó la prueba de desplazamiento del balón con ambos balones (con el balón No 3 de fútbol y con el balón de voleibol). Durante los dos días posteriores se realizaron las tres pruebas de precisión con ambos balones. Los niños que no se encontraban realizando pruebas, realizaron actividades recreativas con la entrenadora.

El remate de precisión, es el acto mediante el cual el jugador efectúa una acción de impulso sobre el balón con la finalidad de acertar en la portería que se encuentra a una distancia alejada. Las pruebas de precisión pueden tener muchas condicionantes, como el clima, el viento, el rebote del balón en el terreno, la percepción y ejecución temporo-espacial adecuada del gesto. Por esta razón, se realizaron tres pruebas de precisión (las cuales contaban con diez intentos cada una - cinco con cada balón) y una de desplazamiento (cinco intentos con cada balón). Para las pruebas de precisión los participantes se posicionaban en el terreno de juego, con el balón ubicado a una distancia entre $8 \mathrm{~m}$ y $10 \mathrm{~m}$ de la portería en una zona delimitada. El balón se encontraba estático y centrado al punto medio de la línea de portería a la hora de llevarse a cabo la ejecución. Los resultados de los remates se registraron en una planilla, indicando con un número 1 cuando se convertía y número zero cuando no se convertía el gol. Para validar la conversión se tomaron en cuenta las reglas oficiales del fútbol según ONFI (Organización Nacional de Fútbol Infantil, 2017). Las pruebas son descritas abajo:

\section{Prueba de precisión con portería reglamentaria de fútbol para niños:}

La portería era rectangular, de $4 \mathrm{~m}$ de longitud entre postes y $2 \mathrm{~m}$ de altura desde el borde inferior del travesaño al suelo. El objetivo de la prueba era convertir el gol. Además, Se registró en la planilla por qué zona ingresaba el balón, indicando con una letra $D$, cuando el balón ingresaba por debajo de la línea media horizontal de la portería y con una letra $U$, cuando ingresaba por arriba.

\section{II) Prueba de precisión en mini portería:}

El objetivo de la prueba era convertir el gol en la mini portería. Las medidas de la mini portería eran: $78 \mathrm{~cm}$ altura $\times 56 \mathrm{~cm}$ ancho $\times 45 \mathrm{~cm}$ profundidad.

\section{III) Prueba de precisión en portería reglamentaria de fútbol para niños, con obstáculo:}

Los niños se aproximaban al balón y remataban. Había una portería de fútbol para niños con un obstáculo ubicado en el centro y en el suelo de la misma. Debían convertir en los espacios que dejaba libre el obstáculo dentro de la portería. Cada remate contó como un intento. El objetivo de la prueba era convertir el gol, evitando el obstáculo.

Prueba de desplazamiento del balón:

Otro de los factores claves para un buen remate, además de la precisión, es la fuerza aplicada sobre el balón en el momento del impacto. Para evaluar el efecto de dicha fuerza se hizo una única prueba, donde se midió el desplazamiento del balón luego del remate. Para la realización de esta prueba se solicitó a los participantes que remataran con toda su fuerza y de forma aérea y se midió la distancia recorrida por el balón hasta el primer impacto con el suelo.

El individuo se posicionaba al costado de la portería, fuera del terreno de juego. El balón se encontraba de forma estática en uno de los vértices de la línea del área de meta, a $4 \mathrm{~m}$ de la misma. A la hora de llevarse a cabo la ejecución, el individuo se aproximaba iniciando su carrera desde la línea de meta hacia el balón y remataba de tal forma que el balón impactara con el suelo lo más lejos posible. La distancia que recorría el balón, desde el punto de inicio, hasta el primer impacto en el terreno de juego, fue registrada en una planilla por el investigador responsable. Había líneas que marcaban la distancia cada un metro desde el punto de donde partía el balón. 


\section{ESTADÍSTICA}

Los datos son presentados a través de media y desviación standard. Para el análisis de los datos obtenidos en cada una de las pruebas se utilizó un test de $t$ de medidas pareadas con un nivel de significancia $p \leq 0.05$, dos colas.

\section{RESULTADOS}

Fueron encontradas diferencias entre los balones en la prueba de precisión en portería reglamentaria de fútbol para niños, con obstáculo y de desplazamiento del balón. Los resultados son presentados en la tabla 1.

En la prueba de precisión en portería reglamentaria de fútbol para niños, los remates ejecutados fueron dirigidos debajo de la zona media de la portería. Con el balón de voleibol hubo un total de 56 conversiones de un total de 65 remates ejecutados, de las cuales 46 fueron dirigidas debajo de la línea media horizontal de la portería y 10 fueron dirigidas por arriba de la línea media horizontal de la portería.

En la prueba de precisión en mini portería se observó que con el balón de fútbol hubo un total de 30 conversiones de 60 remates ejecutados. Con el balón de voleibol hubo un total de 19 conversiones de 60 remates ejecutados.

En la prueba de precisión en portería reglamentaria de fútbol para niños con obstáculo, se encontró diferencia entre las medias a favor del balón de voleibol $(P=0,0003)$. Además, se observó que con el balón de fútbol hubo un total de 17 conversiones de 70 remates ejecutados. Con el balón de voleibol hubo un total de 41 conversiones de 70 remates ejecutados.

En la prueba de desplazamiento, con el balón de fútbol se encontraron diferencias significativas entre las medias a favor del balón de voleibol $(P=0,0001)$.

Tabla 1: Presenta los resultados de las diferentes pruebas comparando el balón de fútbol y el balón de voleibol. Pruebas

\begin{tabular}{lcccc}
\hline & $\begin{array}{c}\text { Precis portería } \\
\text { reglamentaria }\end{array}$ & $\begin{array}{c}\text { Precis mini portería } \\
\text { obstáculo }\end{array}$ & $\begin{array}{c}\text { Desplaz máx aéreo } \\
(\mathrm{m})\end{array}$ \\
\hline Conversiones Fút & $4,07 \pm 0,86$ & $2,5 \pm 1,38$ & $1,21 \pm 0,95$ & --- \\
Conversiones Vól & $4,3 \pm 0,63$ & $1,58 \pm 0,79$ & $2,93 \pm 1,15$ & --- \\
Desplazamiento Fút & --- & --- & --- & $6,46 \pm 2,75$ \\
Desplazamiento Vól & --- & --- & --- & $9,1 \pm 3,43$ \\
P valor & 0,33 & 0,12 & 0,0003 & 0,0001 \\
\hline
\end{tabular}

Los valores son presentados en media y desviación standard. Prueba de precisión en portería reglamentaria de fútbol para niños con obstáculo, prueba de precisión en mini portería, prueba de precisión con portería reglamentaria de fútbol para niños, desplazamiento máximo aéreo, conversiones con balón de fútbol, conversiones con balón de voleibol, desplazamiento con balón de fútbol, desplazamiento con balón de voleibol.

\section{DISCUSIÓN}

Se comprueba parcialmente la hipótesis, el balón de voleibol fue más adecuado que el balón de fútbol para la precisión en portería reglamentaria de fútbol para ninõs con obstáculo y el desplazamiento aéreo máximo con el gesto de remate.

En prueba de precisión en portería reglamentaria de fútbol para niños, se observa que el balón de voleibol no produjo una diferencia en la precisión del remate en comparación con el balón de fútbol. Esto puede deberse a que el nivel de dificultad en esta prueba no era muy complejo y los niños lograron convertir con facilidad con ambos balones. El nivel motor, en general, para niños con la edad involucrada en esta investigación, está preparado para realizar el tipo de acciones motrices propuestas esta prueba sin inconvenientes. Como expresan González y Rada de Rey, el desarrollo motor grueso en esta etapa incluye el rematar y correr como una actividad que no conlleva la pesadez que ejercía años anteriores. (González y Rada de Rey, 1997). Al poseer los niños estas cualida- 
des, pudieron realizar esta prueba sin mayores dificultades con ambos balones, y sin tener ningún impedimento causado por el peso de los mismos.

A pesar de esto, sí hubo una diferencia en la dirección de los remates al comparar ambos balones, lo que podría deberse a que, los niños no pudieron elevar ninguno de los remates por encima de la línea media de la portería con el balón de fútbol, problablemente por la masa de dicho elemento, y que, es posible que el balón no sea el más adecuado para ésta etapa de formación deportiva. Como ya se mencionó anteriormente haciendo referencia a Meinel y Schnabel, es fundamental que los materiales sean adecuados a la edad madurativa de los niños, para poder avanzar progresivamente en los estímulos y no generar lesiones ni frustraciones (Meinel y Schnabel, 2004).

En la prueba de precisión en mini portería, al igual que en la prueba anterior mencionada, se observó que el balón de voleibol no produjo una diferencia en la precisión del remate, en comparación con el balón de fútbol. Además, los niños tenían una cierta adaptación al balón de fútbol por sus prácticas habituales con el mismo, y es posible, que si se hubiera realizado una adaptación al balón de voleibol previa a la prueba, los niños hubieran conseguido convertir un mayor número de intentos con este último balón. Considerando esta afirmación, se toman como referencia las ideas expuestas por González y Rada de Rey en cuanto a las características de adaptación y aprendizaje de los niños (González y Rada de Rey, 1997) y se matiza también en lo expresado por Castañer y Camerino, sobre la capacidad de regular de forma precisa, la intervención del propio cuerpo, en la ejecución de la acción justa y necesaria según la idea motriz prefijada. Los niños, antes de realizar la prueba, poseían una idea prefijada en relación al balón de fútbol por su práctica habitual, que favoreció la conversión con el mismo (Castañer y Camerino, 2001).

En la prueba de precisión en portería reglamentaria de fútbol para niños con obstáculo, se observó que el balón de voleibol produjo una diferencia en la precisión del remate en comparación con el balón de fútbol. En esta prueba, que presenta un mayor grado de complejidad que las anteriores, se observó que resulta más fácil para los niños, direccionar y evitar el obstáculo, con el balón de voleibol que con el reglamentario, indicando esto, la mayor adecuación que presenta el balón más liviano en esta etapa de la niñez para el remate de precisión.

En la prueba de desplazamiento del balón, se observó que el balón de voleibol, produjo una diferencia en el desplazamiento del remate en comparación con el balón de fútbol. Como ya se mencionó, uno de los factores más importantes para el remate, es la fuerza que se puede aplicar en dicho gesto, y la reacción que tenga el balón hacia esa fuerza aplicada, está directamente relacionada con la masa del elemento. Los resultados de esta prueba, manifiestan que el balón de voleibol permitió lograr mayores distancias que el balón de fútbol, en cuanto a la fuerza que los niños pueden aplicarle en el remate de desplazamiento máximo.

\section{Limitaciones del estudio}

Los niños, antes de realizar las pruebas, poseían experiencia de práctica solamente con el balón de fútbol y no con el de voleibol por lo que conocían mejor uno que el otro.

Más investigaciones a respecto del tema, con análisis cinemático, diferentes situaciones de juego son importantes.

\section{CONCLUSIÓN}

Nuestra hipótesis fue parcialmente comprobada. Fueron encontrados mejores resultados en remates de precisión en portería reglamentaria de fútbol para niños con obstáculo y desplazamiento máximo para dicha muestra. No se manifiesta aquí cuál es el balón óptimo y exacto en fútbol, ya que dicha tarea, requeriría la fabricación de un elemento específico y acorde a las características puntuales de la muestra de niños estudiada. Pero pensar en materiales adecuados para el aprendizaje y la práctica deportiva en esta edad es importante.

\section{APLICACIÓN PRÁCTICA}

Sería de esencial importancia para la formación de base deportiva, el seguir inquiriendo en la búsqueda del balón de fútbol más adecuado para el periodo de la niñez estudiado en esta ocasión. Además, es posible 
que otros elementos y espacios físicos empleados en fútbol para niños no sean acorde a las facultades de ellos, quedando un gran margen de investigación hacia la búsqueda y optimización de dichos recursos. Es importante evitar en los niños posibles lesiones, dicho cuidado en la formación, demanda la implementación de los correctos estímulos pedagógicos y didácticos, siendo parte fundamental aquí, los materiales utilizados en el proceso de aprendizaje.

\section{REFERENCIAS}

BACKOUS, D. D.; FRIEDL, K. E.; SMITH, N. J.; PARR, T. J., \& CARPINE; W. D. (1988). Soccer injuries and their relation to physical maturity. American Journal of Diseases of Children, 142(8), 839-842.

BABKES, M. L.; \& WEISS, M. R. (1999). Parental influence on children's cognitive and affective responses to competitive soccer participation. Pediatric Exercise Science, 11(1), 44-62.

BENEDEK, E. (2006). Fútbol infantil. España: Paidotribo. CASTAÑER, M., \& CAMERINO, O. (2001). La Educación Física En La Educación Primaria. España: INDE.

CASTELO, J. (1999). Fútbol: Estructura y dinámica del juego. España: INDE.

GONZÁLEZ, M. L.; \& RADA DE REY, B. A. (1997). La educación física y su didáctica. Argentina: A-Z.
LEES, A.; ASAI, T.; ANDERSEN, T. B.; NUNOME, H.; \& STERZING, T. (2010). The biomechanics of kicking in soccer: A review. Journal of sports sciences, 28(8), 805-817.

LEVY, M. L.; KASASBEH, A. S.; BAIRD, L. C.; AMENE, C., SKEEN, J. \& MARSHALL; L. (2012). Concussions in soccer: a current understanding. World neurosurgery, 78(5), 535-544.

MEINEL, K. \& SCHNABEL, G. (2004). Teoría del movimiento. Argentina: Stadium.

PADUA, D. A.; DISTEFANO, L. J.; MARSHALL, S. W.; BEUTLER, A. I.; DE LA MOTTE, S. J. \& DISTEFANO, M. J. (2012). Retention of movement pattern changes after a lower extremity injury prevention program is affected by program duration. The American journal of sports medicine, 40(2), 300-306.

O'KANE, J. W.; SPIEKER, A.; LEVY, M. R.; NERADILEK, M.; POLISSAR, N. L., \& SCHIFF, M. A. (2014). Concussion among female middle-school soccer players. JAMA pediatrics, 168(3), 258-264.

ORGANIZACIÓN NACIONAL DE FÚTBOL INFANTIL (ONFI). (2017). Reglas de Juego. Montevideo: http://www.onfi.org. uy/onfi_mixto/index.php/reglamentos/1164-reglas-juego-2017.

RÖSSLER, R.; JUNGE, A.; CHOMIAK, J.; DVORAK, J.; \& FAUDE, O. (2016). Soccer injuries in players aged 7 to 12 years: a descriptive epidemiological study over 2 seasons. The American journal of sports medicine, 44(2), 309-317.

VERBURGH, L.; SCHERDER, E. J.; VAN LANGE, P. A.; \& OOSTERLAAN, J. (2014). Executive functioning in highly talented soccer players. PloS one, 9(3), e91254. 